\title{
Análise bibliométrica da produção científica utilizando microtomografia computadorizada apresentada nas reuniões anuais da Sociedade Brasileira de
}

\section{Pesquisa Odontológica}

\author{
Bibliometric analysis of the scientific production using computed microtomography presented at
}

the Brazilian Society of Dental Research annual meetings

Análisis bibliométrico de la producción científica mediante microtomografía computarizada

presentada en las reuniones anuales de la Sociedad Brasileña de Investigación Odontológica

Recebido: 16/03/2021 | Revisado: 22/03/2021 | Aceito: 29/03/2021 | Publicado: 08/04/2021

Moan Jéfter Fernandes Costa

ORCID: https://orcid.org/0000-00002-7250-5863

Universidade de Pernambuco, Brasil

E-mail: moanjefter@gmail.com

Alice Castro Guedes Mendonça

ORCID: https://orcid.org/0000-0003-1572-7547 Universidade Federal da Paraíba, Brasil

E-mail: alicecgm@hotmail.com

Leonardo de Freitas Ferreira

ORCID: https://orcid.org/0000-0002-4948-4347

Universidade Federal da Paraíba, Brasil E-mail: leoffferreira@gmail.com

Hugo Victor Dantas

ORCID: https://orcid.org/0000-0002-3931-0802 Universidade Federal da Paraíba, Brasil E-mail: hugodantas92@gmail.com

Basílio Rodrigues Vieira

ORCID: https://orcid.org/0000-0003-2025-7773

Faculdade São Francisco da Paraíba, Brasil E-mail: basilio_451@hotmail.com

Eugenia Livia de Andrade Dantas ORCID: https://orcid.org/0000-0003-2344-4213

Universidade Federal da Paraíba, Brasil E-mail: eugenialivia@ @otmail.com

\begin{abstract}
Resumo
A microtomografia computadorizada utiliza o princípio de raios-x para formação de imagens bi e tridimensionais de amostras pequenas com alta resolução. O encontro anual da Sociedade Brasileira de Pesquisa Odontológica (SBPqO) é o maior evento de produção científica em Odontologia do país. O perfil dos trabalhos expostos pode identificar características da pesquisa científica produzida nacionalmente. O objetivo deste estudo foi analisar retrospectivamente a produção científica envolvendo microtomografia computadorizada (Micro-CT) nos suplementos das reuniões do SBPqO de 2009 a 2018. Após leitura dos anais de todos os anos utilizando os descritores "Microtomografia Computadorizada", "Micro-CT", "MicroCT", "mCT", "mTC" e " $\mu \mathrm{CT}$ ", a amostra final foi um total de 348 estudos utilizando Micro-CT. O ano com maior número de resumos foi $2016(22,7 \%$; n=79). Os estudos foram mais frequentes na região Sudeste $(86,2 \% ; n=300)$, com mais estudos na área de endodontia $(35,0 \% ; n=122)$, sendo os elementos dentários humanos mais utilizados como amostras $(53,7 \% ; \mathrm{n}=187)$. Houve aumento no número de pesquisas envolvendo Micro-CT, mas houve maior concentração para a região Sudeste e para a área de endodontia. Assim, destaca-se a necessidade de disseminação do conhecimento para que outras áreas também possam utilizar essa ferramenta e aumentar o escopo da produção odontológica.
\end{abstract}

Palavras-chave: Microtomografia por Raio-X; Pesquisa; Odontologia.

\section{Abstract}

Computed microtomography uses the principle of $\mathrm{x}$-rays to form two- and three-dimensional images of small samples with high resolution. The annual meeting of the Brazilian Society of Dental Research is the largest event of scientific production in Dentistry in the country. The profile of the papers exhibited can identify characteristics of the scientific research produced nationally. The aim of this study was to retrospectively analyze the scientific production involving 
micro computed tomography (Micro-CT) in the supplements of SBPqO meetings from 2009 to 2018. After reading the annals of all years using the descriptors "Computed Microtomography", "Micro-CT", "MicroCT", "mCT", "mTC" and " $\mu \mathrm{CT}$ ", the final sample was a total of 348 studies using Micro-CT. The year with the highest number of abstracts was $2016(22.7 \% ; n=79)$. The studies were most frequent in the southeastern region $(86.2 \% ; n=300)$, that presented the most studies were Endodontics $(35.0 \%$; $\mathrm{n}=122)$ and the most sample used human dental elements $(53.7 \% ; \mathrm{n}=187)$. There was an increasing increase in the number of research involving Micro-CT, but there was a greater concentration for the Southeast region and in the area of endodontics. Thus, the need for knowledge dissemination is highlighted so that other areas can also use this tool and increase the scope of dental production.

Keywords: Microtomography by X-Ray; Research; Dentistry.

\section{Resumen}

La microtomografía computarizada utiliza el principio de los rayos $\mathrm{X}$ para formar imágenes bidimensionales y tridimensionales de pequeñas muestras con alta resolución. La reunión anual de la Sociedad Brasileña de Investigación Odontológica es el mayor evento de producción científica en Odontología en el país. El perfil de los trabajos expuestos puede identificar características de la investigación científica producida nacionalmente. El objetivo de este estudio fue analizar retrospectivamente la producción científica que involucra la tomografía microcomputada (Micro-CT) en los suplementos de las reuniones de la SBPqO desde 2009 hasta 2018. Después de leer los anales de todos los años utilizando los descriptores "Microtomografía computarizada", "Micro-CT", "MicroCT", "mCT", "mTC" y " $\mu \mathrm{CT}$ ", la muestra final fue de un total de 348 estudios con Micro-CT. El año con mayor número de resúmenes fue 2016 (22,7\%; n=79). Los estudios fueron más frecuentes en la región sureste $(86,2 \%$; n=300), los que más estudios presentaron fueron de Endodoncia $(35,0 \% ; \mathrm{n}=122)$ y la mayor muestra utilizó elementos dentales humanos $(53,7 \% ; \mathrm{n}=187)$. Hubo un aumento en el número de investigaciones que involucran la Micro-TC, pero hubo una mayor concentración para la región Sudeste y en el área de endodoncia. Así, se destaca la necesidad de difusión del conocimiento para que otras áreas también puedan utilizar esta herramienta y aumentar el alcance de la producción odontológica.

Palabras clave: Microtomografia por Rayos X; Investigación; Odontología.

\section{Introdução}

A Microtomografia Computadorizada por raios-X (Micro-CT ou $\mu \mathrm{CT}$ ) foi desenvolvida na década de 80 com propriedades melhoradas quando comparada à Tomografia Computadorizada por raios-X (TC), apresentando voxels menores em volume, o que resulta em melhor resolução espacial (Feldkamp et al., 1989; Kuhn et al., 1990). O equipamento é um componente essencial de muitos laboratórios de pesquisa acadêmica e industrial. Seus recursos são muito utilizados na Odontologia para geração de modelos tridimensionais de elementos finitos e para examinar tecidos mineralizados e materiais dentários (Verdonschot et al., 2001; Costa et al., 2014; El-Wassefy 2017; Lee et al., 2017, Palhais et al., 2017; Zhang et al., 2017). O seu uso tem se destacado em algumas áreas odontológicas, tais como a Endodontia, Reabilitação Oral, Periodontia e Cariologia (Verdonschot et al., 2001; Costa et al., 2014; ElWassefy 2017; Lee et al., 2017, Palhais et al., 2017; Zhang et al., 2017).

A tecnologia permite pesquisas in vivo com pequenos animais e demonstra resultados comparáveis à análise histológica (Xia et al., 2016; Pi et al., 2017). Seu uso na Odontologia passou a ser de fundamental importância para a formação de imagens em pequena escala com aumento exponencial da resolução. É caracterizada por ser uma metodologia não invasiva, sem necessitar de um processo complexo para a avaliação dos espécimes, proporcionando visualização tridimensional interna do material em estudo (Palhais et al., 2017; Zhang et al., 2017).

Os estudos mostram que a progressão das descobertas da pesquisa básica é fundamental para a evolução da clínica e para a implementação dos resultados nos processos de saúde. A aprendizagem baseada no desenvolvimento de pesquisas, no levantamento de problemas e na formulação de hipóteses pode levar ao pensamento crítico e a avanços na Odontologia (Slavkin 2017).

Com relação às pesquisas realizadas com Micro-CT não é diferente, ela tem sido vista como uma ferramenta poderosa em futuras pesquisas odontológicas e são amplamente utilizados em muitos campos acadêmicos (Swain \& Xue 2009). Para entender a realidade atual desta ferramenta, o objetivo deste estudo foi avaliar o uso da Micro-CT em pesquisas odontológicas apresentadas no maior evento científico nacional em odontologia do Brasil. 


\section{Metodologia}

Foi realizado um estudo observacional retrospectivo por meio da análise dos resumos publicados nos anais da Sociedade Brasileira de Pesquisa Odontológica (SBPqO), braço brasileiro da International Association for Dental Research (IADR), no período de 10 anos (2009 a 2018; com exceção de 2012, ano em que o IADR aconteceu no Brasil), em todas as categorias disponíveis nos anais publicados em http://www.sbpqo.org.br.

Para a coleta de dados foram utilizados marcadores, na qual a busca foi feita separadamente entre os termos "Microtomografia Computadorizada", "Micro-CT", "MicroCT", "mCT", "mTC" e " $\mu C T$ ", com a extração dos dados realizada por dois avaliadores (MJFC e ELAD) de forma independente. Em casos de discordância, este foi avaliado por um terceiro examinador (BRV), como forma de haver um consenso entre os examinadores.

Antes da seleção oficial dos resumos, os avaliadores foram treinados para a revisão de conceitos e parâmetros relacionados ao estudo visando à estandardização na conduta da coleta de dados e à padronização de avaliação e foi gerado um Kappa de 0.91, após a avaliação de 30 resumos. Os resumos foram avaliados quanto à presença dos descritores no título, nas palavras-chaves ou inseridos no texto do resumo, de forma que fosse constatado que o trabalho em foco teria utilizado a Micro-CT em alguma das etapas da pesquisa.

Pelo fato da Reunião anual da SBPqO ser um congresso multidisciplinar tem-se a interação entre trabalhos das mais diversas áreas da Odontologia. As especialidades foram agrupadas de acordo com a proximidade de assuntos em: Endodontia (instrumentos, materiais e anatomia interna), Periodontia, Cirurgia (anatomia topográfica, Implantodontia, Farmacologia e Anestesiologia), Patologia (Radiologia e Diagnóstico Oral), Reabilitação Oral (Dentística, Prótese, Materiais Dentários, Cariologia, Oclusão e Disfunção Temporomandibular), Ortodontia (Ortodontia e Odontopediatria) e docência (instrumentos de ensino).

Para categorização dos resumos, além da separação por ano de publicação, foram observados a região que foi realizada o estudo (de acordo com a instituição de origem mencionada no resumo), a área temática e o tipo de amostra analisada. Os dados foram organizados e analisados por meio de estatísticas descritivas e gráficos no software Microsoft Office Excel 2019.

\section{Resultados}

Um total de 27582 trabalhos foi obtido no período avaliado. Destes, 348 utilizaram a Micro-CT, 79 no ano de 2016, totalizando 22,7\% dos trabalhos avaliados, sendo o ano com maior percentual durante os anos analisados (Gráfico 1).

Gráfico 1. Total de resumos publicados nos anais nos anos de 2009 a 2018

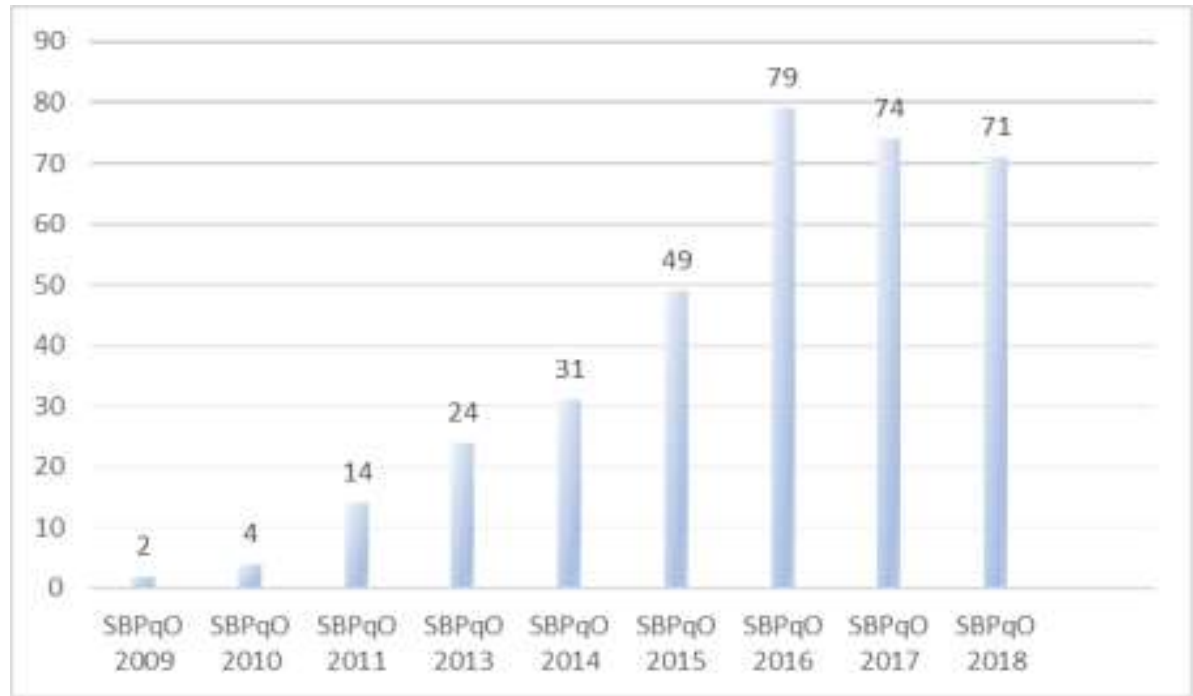

Fonte: Autores. 
Na avaliação da distribuição geográfica, a região do país com maiores taxas de concentração de pesquisa utilizando a MicroCT foi a região Sudeste $(n=301 ; 86,2 \%)$, seguida pelas regiões Sul $(n=26 ; 7,4 \%)$ e Nordeste $(n=16 ; 4,6 \%)$, sendo encontrados apenas dois estudos na região Norte $(\mathrm{n}=2 ; 0,5 \%)$ (Gráfico 2$)$.

Gráfico 2 - Total de resumos publicados nos anais por região entre 2009 e 2018.

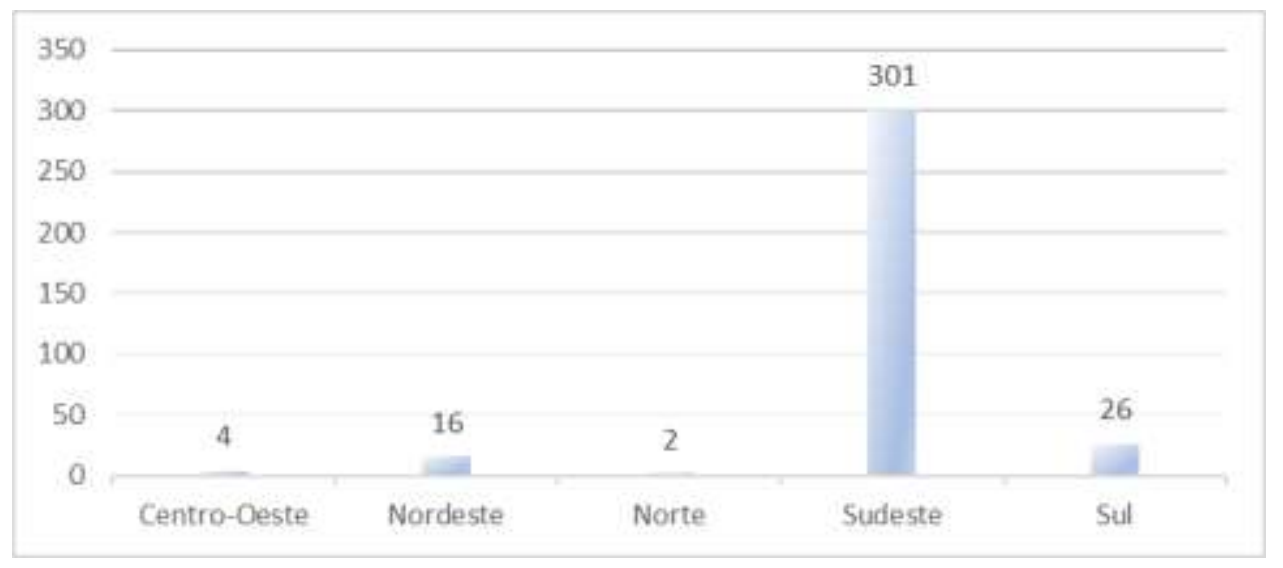

Fonte: Autores.

Quando avaliada a utilização da técnica por área temática, observou-se que a Endodontia apresenta o maior percentual ( $\mathrm{n}=301 ; 35,0 \%)$, seguida pela Reabilitação Oral $(\mathrm{n}=110 ; 28,7 \%)$ e Patologia $(\mathrm{n}=48 ; 13,8 \%)$. As demais áreas temáticas estão expostas no Gráfico 3.

Gráfico 3 - Total de resumos publicados nos anais por área de estudo entre 2009 e 2018.

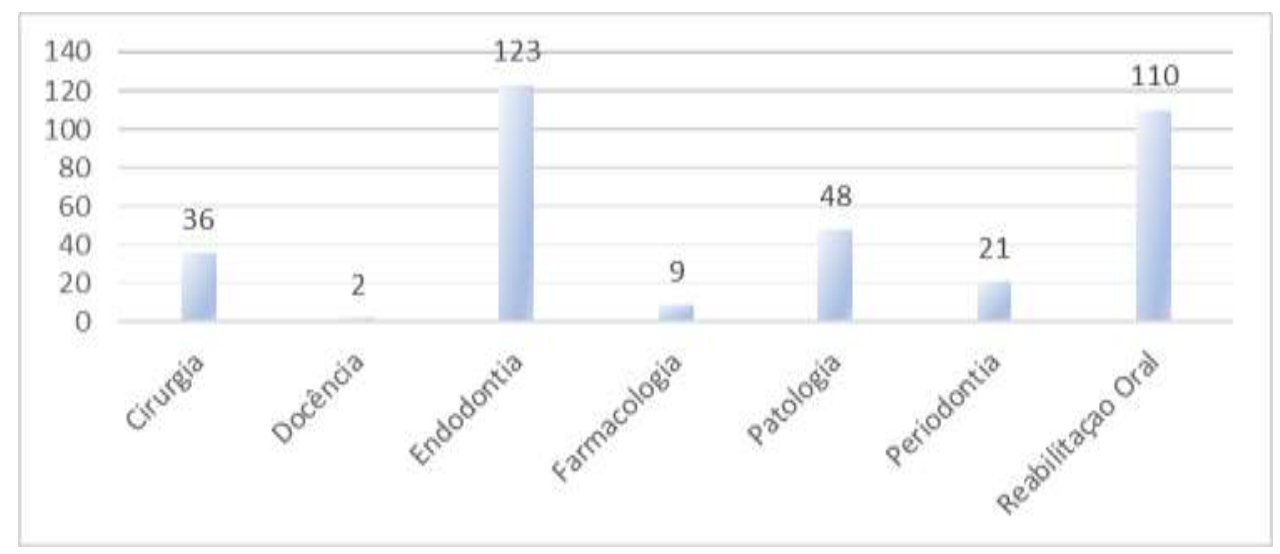

Fonte: Autores.

Os elementos dentais humanos foram a maioria $(n=188 ; 53,7 \%)$ das amostras analisadas (Gráfico 4). Porém, amostras como elementos dentais bovinos, peças ósseas de animais e materiais dentários também foram estudados. 
Gráfico 4 - Total de resumos publicados nos anais de acordo com as diferentes amostras utilizadas para estudo entre 2009 e 2018

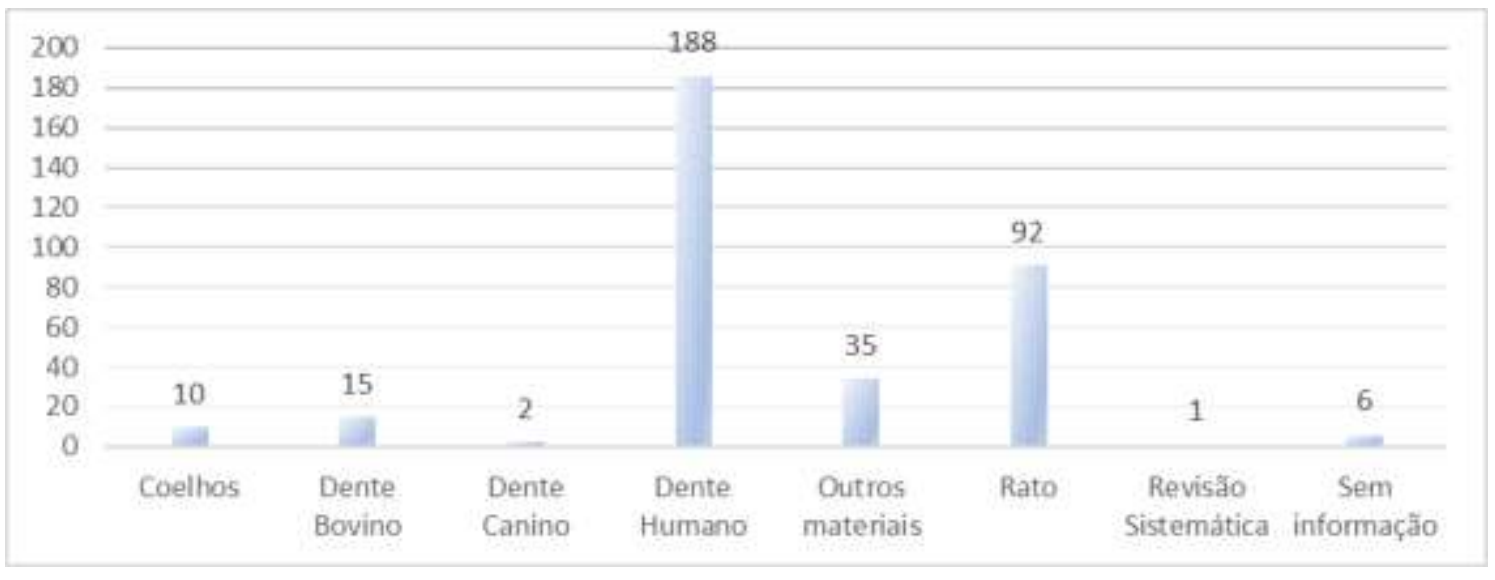

Fonte: Autores.

\section{Discussão}

O uso da Micro-CT na odontologia tornou-se importante pela análise em pequena escala com aumento exponencial de resolução e principalmente por permitir a análise não invasiva de amostras com fácil processo de preparo (Palhais et al., 2017; Zhang et al., 2017). A microscopia confocal permite a obtenção de imagens de alta resolução por meio de cortes ópticos, posteriormente agrupados para se fazer a reconstrução tridimensional da topografia de objetos complexos. Outra técnica que também permite a obtenção de imagens (polidas ou rugosas) em alta resolução com grande profundidade de campos e alta resolução é a microscopia eletrônica de varredura (MEV). Ambas as técnicas são amplamente utilizadas na Odontologia métodos de caracterização de materiais odontológicos em pesquisas (Queiroz et al., 2012), porém quando comparadas à Micro-CT apresentam a desvantagem de serem análises destrutivas.

A reunião anual da $\mathrm{SBPqO}$, maior evento nacional de pesquisas em Odontologia, evidencia em seus anais que ao longo dos anos a pesquisa com Micro-CT tem aumentado nas diversas regiões do país e áreas de estudo. Percebe-se aumento considerável entre os anos de 2009 e 2016. Essa constatação corrobora o fato de que o Brasil estava, em 2015, em segundo lugar no ranking de produções científicas mundiais, representando quase $7 \%$ de toda a produção científica dos últimos anos, atrás apenas dos Estados Unidos (Gracio et al., 2013; Normando 2014).

Com relação às regiões que mais publicam resumos nos anais da $\mathrm{SBPqO}$, a região "Sudeste" domina consideravelmente o campo de pesquisas usando Micro-CT, seguindo a mesma tendência apresentada por outros trabalhos que analisaram a produção científica odontológica brasileira (Barbosa et al., 2019; Gabardo et al., 2019). A região "Sudeste” concentra os principais cursos de pós-graduação com altas notas de avaliação e laboratórios com mais infraestrutura, o que facilita o acesso ao equipamento. Porém, o equipamento apresenta alto custo, exige experiência e conhecimento aprofundado para operá-lo adequadamente (Neto et al., 2011).

A endodontia foi a especialidade que apresentou maior quantidade de trabalhos relacionados com a Micro-CT. Essa técnica não-destrutiva está sendo usada como uma ferramenta precisa para avaliação quantitativa em três dimensões (Freire et al., 2015). As imagens geradas pela Micro-CT revolucionaram a forma de estudar a complexa anatomia interna dos canais radiculares. Além disso, na endodontia é possível distinguir com precisão materiais de preenchimento de espaços vazios e estruturas dentárias (Sousa-Neto, et al., 2018).

A reunião da $\mathrm{SBPqO}$ é um evento multidisciplinar, de forma que ocorre interação entre trabalhos das mais diversas áreas da Odontologia utilizando a Micro-CT. As pesquisas não focam apenas no componente quantitativo de intervenção, mas analisam 
também a qualidade dos trabalhos que estão sendo publicados, a exemplo das imagens formadas a partir da Micro-CT para fornecer melhores condições de entendimento e aprendizagem.

A pesquisa na Odontologia permite o estudo com diversos tipos de espécimes devido às proximidades anatômicas, morfológicas e estruturais com os elementos dentários para que os resultados encontrados permitam inferências à rotina clínica (Swain \& Xue 2009). Ainda assim, nesse estudo, o componente "dentes humanos" segue sendo como mais estudado, de forma a simplificar os valores apresentados. Esse componente engloba tanto o dente completo, quanto a análise das raízes, superfícies proximais ou apenas dos canais radiculares.

Estudos com modelo animal aparecem com frequência representativa nesse estudo, segundo tipo que mais fornece material para a formação de amostras, esses componentes englobam tanto os elementos dentários de ratos como a análise de partes ósseas (calvária, fêmur e tíbia). A variável "outros materiais" trata de estudos com dentes de acrílico, troquéis de resina, coroas de porcelana, implantes de titânio, cimentos endodônticos e resinas.

Por levar em consideração apenas os resumos publicados, podemos referir como limitação deste estudo o fato de não ser possível obter todas as informações de algumas pesquisas incluídas, levando a criação da categoria "sem informação" para os resumos que não exibem as descrições das amostras analisadas. Estudos com Micro-CT se mostram promissores nas diversas áreas da Odontologia, uma vez que apresentam alta qualidade de imagem, possibilidade de modelos didáticos no campo do ensino e podem ser associados com outros métodos de pesquisa, uma vez que a análise não é destrutiva.

\section{Conclusão}

Apesar da evolução no número de pesquisas envolvendo Micro-CT, estas ainda estão concentradas na região Sudeste e na área de endodontia. Assim, em se tratando de Brasil, ainda se destaca de disseminação do conhecimento e de mais investimentos na pesquisa odontológica brasileira para aumentar o escopo da pesquisa no campo odontológico dentro das diversas áreas.

\section{Referências}

Barbosa, L. C., Saliba, T. A., Garbin, C. A. S., \& Moimaz, S. A. S. (2019) Panorama de pesquisas odontológicas brasileiras apresentadas em reunião científica-SBPqO. Rev Odontol UNESP. 48(1):1-9.

Costa, P. F., Vaquette, C., Zhang, Q., Reis, R. L., Ivanovski, S., \& Hutmacher, D. W. (2014). Advanced tissue engineering scaffold design for regeneration of the complex hierarchical periodontal structure. Journal of clinical periodontology,41(3), 283-294. https://doi.org/10.1111/jcpe.12214.

El-Wassefy N. A. (2017). Remineralizing effect of cold plasma and/or bioglass on demineralized enamel. Dental materials journal, 36(2), 157-167. https://doi.org/10.4012/dmj.2016-219.

Feldkamp, L. A., Goldstein, S. A., Parfitt, A. M., Jesion, G., \& Kleerekoper, M. (1989). The direct examination of three-dimensional bone architecture in vitro by computed tomography. Journal of bone and mineral research: the official journal of the American Society for Bone and Mineral Research, 4(1), 3-11.https://doi.org/10.1002/jbmr.5650040103.

Freire, L. G., Iglecias, E. F., Cunha, R. S., Dos Santos, M., \& Gavini, G. (2015). Micro-Computed Tomographic Evaluation of Hard Tissue Debris Removal after Different Irrigation Methods and Its Influence on the Filling of Curved Canals. Journal of endodontics, 41(10), 1660 1666. https://doi.org/10.1016/j.joen.2015.05.001.

Gabardo, M. C. L., Copelli, F. A., Tuzzi, A. L., Trentin, G., Lima, J., Tomazinho, F. S. F., \& Sousa, Y. T. C. S. (2019) Pesquisa científica em Endodontia apresentada na Reunião Anual da Sociedade Brasileira de Pesquisa Odontológica: análise bibliométrica de 2010 a 2018. Rev ABENO. 19(3):144-52.

Gracio, M. C. C., de Oliveira, E. F. T., de Araujo-Gurgel, J., Escalona, M. I., \& Guerrero, A. P. (2013) Dentistry scientometric analysis: a comparative study between Brazil and other most productive countries in the area. Scientometrics. 95(2):753-69.

Kuhn, J. L., Goldstein, S. A., Feldkamp, L. A., Goulet, R. W., \& Jesion, G. (1990). Evaluation of a microcomputed tomography system to 
study trabecular bone structure. Journal of orthopaedic research: official publication of the Orthopaedic Research Society, 8(6), 833-842. https://doi.org/10.1002/jor. 110008060'ç8.

Lee, D. H., Li, L. J., Mai, H. N., Kim, K. R., \& Lee, K. W. (2017). The Effect of a CAD/CAM-Guided Template on Formation of the ScrewAccess Channel for Fixed Prostheses Supported by Lingually Placed Implants. The International journal of prosthodontics, $30(2), 113-115$. https://doi. org/10. 11607/ijp. 4979.

Neto, J. M. R., Fiori, A. P., Lopes, A. P., Marchese, C., Pinto-Coelho, C. V., \& Vasconcellos, E. M. G. (2011) A microtomografia computadorizada de raios x integrada à petrografia no estudo tridimensional de porosidade em rochas. Rev Bras Geociênc. 41(3):498-508.

Normando D. (2014). The Brazilian dental science. Dental press journal of orthodontics, 19(2), 14. https://doi. org/10.1590/2176-9451.19. 2.014-014.edt.

Palhais, M., Sousa-Neto, M. D., Rached-Junior, F. J., Amaral, M. C., Alfredo, E., Miranda, C. E., \& Silva-Sousa, Y. T. (2017). Influence of solvents on the bond strength of resin sealer to intraradicular dentin after retreatment. Brazilian oral research,31, e11. https://doi.org/10.1590/1807-3107BOR-2017. vol31. 0011.

Pi, S., Choi, Y. J., Hwang, S., Lee, D. W., Yook, J. I., Kim, K. H., \& Chung, C. J. (2017). Local Injection of Hyaluronic Acid Filler Improves Open Gingival Embrasure: Validation Through a Rat Model. Journal of periodontology, 88(11), 1221-1230. https://doi.org/10.1902/jop. 2017. 170101.

Queiroz, J. R. C., Marocho, S. S., Benetti, P., Tango, R. N., \& Junior, L. N. (2012). Métodos de caracterização de materiais para pesquisa em odontologia. RFO UPF. 17(1):106-12.

Slavkin H. C. (2017). The Impact of Research on the Future of Dental Education: How Research and Innovation Shape Dental Education and the Dental Profession. Journal of dental education, 81(9), eS108-eS127. https://doi.org/10.21815/JDE. 017. 041.

Sousa-Neto, M. D., Silva-Sousa, Y. C., Mazzi-Chaves, J. F., Carvalho, K., Barbosa, A., Versiani, M. A., Jacobs, R., \& Leoni, G. B. (2018). Root canal preparation using micro-computed tomography analysis: a literature review. Brazilian oral research, 32(1), e66. https://doi. org/10.1590/1807-3107bor-2018. vol32. 0066

Swain, M. V., \& Xue, J. (2009). State of the art of Micro-CT applications in dental research. International journal of oral science, 1(4), 177188. https://doi.org/10.4248/IJOS09031.

Verdonschot, N., Fennis, W. M., Kuijs, R. H., Stolk, J., Kreulen, C. M., \& Creugers, N. H. (2001). Generation of 3-D finite element models of restored human teeth using micro-CT techniques. The International journal of prosthodontics, 14(4), 310-315.

Xia, Y., Zhou, P., Wang, F., Qiu, C., Wang, P., Zhang, Y., Zhao, L., \& Xu, S. (2016). Degradability, biocompatibility, and osteogenesis of biocomposite scaffolds containing nano magnesium phosphate and wheat protein both in vitro and in vivo for bone regeneration. International journal of nanomedicine, 11, 3435-3449. https://doi.org/10.2147/IJN. S105645.

Zhang, L., Joubert, C., Bruder, G., Yang, K., Aseel-Fine, A., Jones, K., \& Rafailovich, M. (2017). Effectiveness of X-ray computed microtomography to determine structure-property relationships of Gutta-percha. Dental materials journal, 36(3), 253-259. https://doi. org/10. 4012/dmj. 2015-441. 